\title{
Comparison of different functional prediction scores using a gene-based permutation model for identifying cancer driver genes
}

\author{
Alice Djotsa Nono ${ }^{1}$, Ken Chen ${ }^{2}$ and Xiaoming Liu ${ }^{1,3^{*}}$
}

From The International Conference on Intelligent Biology and Medicine (ICIBM) 2018

Los Angeles, CA, USA. 10-12 June 2018

\begin{abstract}
Background: Identifying cancer driver genes (CDG) is a crucial step in cancer genomic toward the advancement of precision medicine. However, driver gene discovery is a very challenging task because we are not only dealing with huge amount of data; but we are also faced with the complexity of the disease including the heterogeneity of background somatic mutation rate in each cancer patient. It is generally accepted that CDG harbor variants conferring growth advantage in the malignant cell and they are positively selected, which are critical to cancer development; whereas, non-driver genes harbor random mutations with no functional consequence on cancer. Based on this fact, function prediction based approaches for identifying CDG have been proposed to interrogate the distribution of functional predictions among mutations in cancer genomes (eLS 1-16, 2016). Assuming most of the observed mutations are passenger mutations and given the quantitative predictions for the functional impact of the mutations, genes enriched of functional or deleterious mutations are more likely to be drivers. The promises of these methods have been continually refined and can therefore be applied to increase accuracy in detecting new candidate CDGs. However, current function prediction based approaches only focus on coding mutations and lack a systematic way to pick the best mutation deleteriousness prediction algorithms for usage.

Results: In this study, we propose a new function prediction based approach to discover CDGs through a gene-based permutation approach. Our method not only covers both coding and non-coding regions of the genes; but it also accounts for the heterogeneous mutational context in cohort of cancer patients. The permutation model was implemented independently using seven popular deleteriousness prediction scores covering splicing regions (SPIDEX), coding regions (MetaLR, and VEST3) and pan-genome (CADD, DANN, Fathmm-MKL coding and Fathmm-MKL noncoding). We applied this new approach to somatic single nucleotide variants (SNVs) from whole-genome sequences of 119 breast and 24 lung cancer patients and compared the seven deleteriousness prediction scores for their performance in this study.

(Continued on next page)
\end{abstract}

\footnotetext{
* Correspondence: xiaomingliu@health.usf.edu

'Human Genetics Center, UTHealth School of Public Health, Houston, TX, USA

${ }^{3}$ Present Address: USF Genomics, College of Public Health, University of

South Florida, Tampa, FL, USA

Full list of author information is available at the end of the article
}

(c) The Author(s). 2019 Open Access This article is distributed under the terms of the Creative Commons Attribution 4.0 International License (http://creativecommons.org/licenses/by/4.0/), which permits unrestricted use, distribution, and reproduction in any medium, provided you give appropriate credit to the original author(s) and the source, provide a link to the Creative Commons license, and indicate if changes were made. The Creative Commons Public Domain Dedication waiver (http://creativecommons.org/publicdomain/zero/1.0/) applies to the data made available in this article, unless otherwise stated. 


\begin{abstract}
(Continued from previous page)
Conclusion: The new function prediction based approach not only predicted known cancer genes listed in the Cancer Gene Census (CGC), but also new candidate CDGs that are worth further investigation. The results showed the advantage of utilizing pan-genome deleteriousness prediction scores in function prediction based methods. Although VEST3 score, a deleteriousness prediction score for missense mutations, has the best performance in breast cancer, it was topped by CADD and Fathmm-MKL coding, two pan-genome deleteriousness prediction scores, in lung cancer.
\end{abstract}

Keywords: Cancer genomics, Driver genes, Function prediction method, Computational evaluation, Bioinformatics, Whole genome sequencing

\section{Background}

The genetic backgrounds of cancers are highly heterogeneous [1], with almost 719 genes currently known as causally implicated in cancer etiology or development [2, $3]$, some genes are associated with more than one cancer type and this list is far from complete. Over the last decade, due to falling cost of high throughput sequencing, whole genome sequencing analysis has begun to take the place of exome sequencing as the method of choice for investigating genetic variants. It is widely known that in cancer genomics somatic mutations are assumed to occur randomly; however, not all these mutations are involved in carcinogenesis. Pathogenic driver mutations provide growth advantage to cancer cells; whereas, nonpathogenic passenger mutations occurring during tumorigenesis may or may not have functional effect, but play no role in cancer. Cancer driver genes (CDGs) by definition carry at least one driver mutations that increase cell growth advantage. It is challenging to identify signal of positive selection in CDGs that differentiate them for passenger genes harboring only random passenger mutations. Because of the high cost of experimental studies of gene functions, computational predictive algorithms become crucial to assess the evidence of candidate CDGs in a cohort of sequenced cancer samples.

Here we introduce a gene-based permutation model (dubbed Sum of Most Deleterious Score or SMDS) to predict cancer CDGs in light of the pioneering InVeX method [4], a random permutation algorithm. Our algorithm infers enrichment of functional variants at each gene locus (Fig. 1) and applied it to predict the CDGs of breast and lung cancer. Unlike the InVEx approach which utilizes only one functional predictive method for missense SNVs (PolyPhen-2) [5], our algorithm leverages seven different scoring systems through a permutation based model. In addition, this new method covers both coding and non-coding regions of genes in order to infer new CDGs. We assume that for a cancer sample, one pathogenic driver mutation on a CDGs is enough to cause cancer, but different sample may have different pathogenic mutations in different driver genes. This implies that for each driver gene, only a small proportion of the samples may carry driver mutations. The power of our method to detect such driver gene depends on how different the deleteriousness prediction scores of the driver mutations compared to the artificial mutations we randomly imposed on to the gene. Given a list of observed SNVs from a cohort of cancer patients (samples), for each gene, the permutation approach randomly samples the position of each observed SNVs along a given gene sequence maintaining the trinucleotide context. Next, it identifies the Most Deleterious Score (MDS) per gene and sample (a monochromic measure of deleteriousness ranging from 0 to 1 ; and the larger the score, the more likely the variant is deleterious). Then, it tallies for each gene and across patients the SMDS 1000 times to build a null distribution and finally it computes an empirical $p$-value by comparing the observed SMDS against the null distribution of simulated SMDS.

\section{Results}

\section{Comparison of the functional prediction scores}

We have curated somatic mutations data from primary whole genome samples of two cancer types including 278,152 SNVs from 119 breast cancer patients and $468,348 \mathrm{SNVs}$ from 24 lung cancer patients. These individual datasets belong to a large published dataset containing both whole-genome sequencing (WGS) and whole-exome sequencing data (WES) data [6]. After filtering out intergenic SNVs, the functional effect of these SNVs were scored using seven predictive methods: CADD [7], DANN [8], Fathmm-MKL coding and noncoding [9], MetaLR [10], SPIDEX [11] and VEST3 [12]. A summary of the seven predictive scores is presented in Additional file 1: Table S1.

First, we used the pairwise Pearson correlation coefficient $(r)$ to measure the relationship between any pairs of the seven scores (Additional file 1: Table S2). Two pairs of scores (Fathmm-MKL coding and Fathmm-MKL noncoding, MetaLR and VEST3) were highly correlated $(r \geq 0.7)$; while six pairs of scores show medium correlation $(0.4<r$ 
SNVs in each gene $\mathbf{\nabla}=$ Single Nucleotide Variants (SNVs) in gene $g_{j} \quad \boldsymbol{\nabla}=$ Most deleterious SNV *
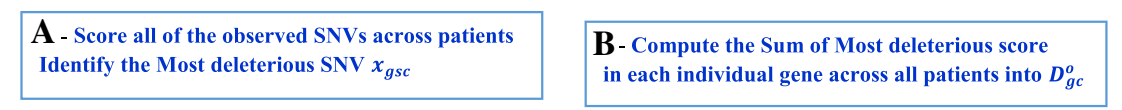

E- Compute the p-value of $D_{g c}^{o}$ for Gene 1 based on the distribution of $1000 D_{g c}^{m}$
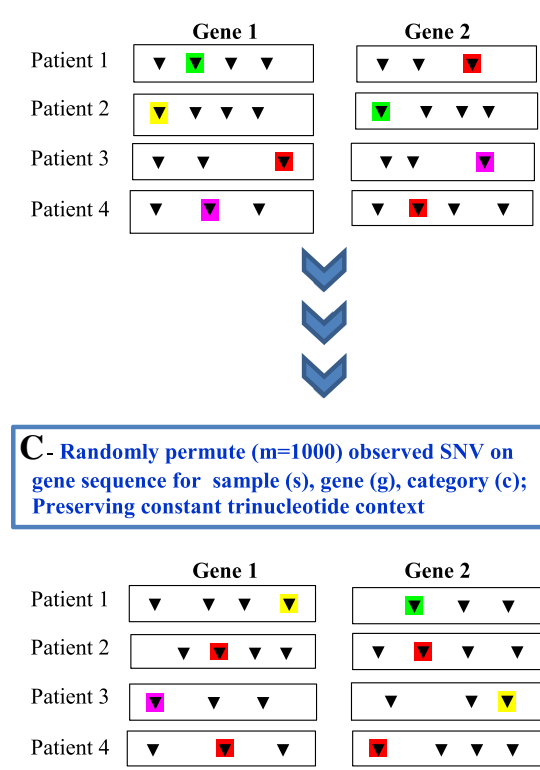

observed SNV on gene sequence for sample (s), gene (g), category (c); Preserving constant trinucleotide context

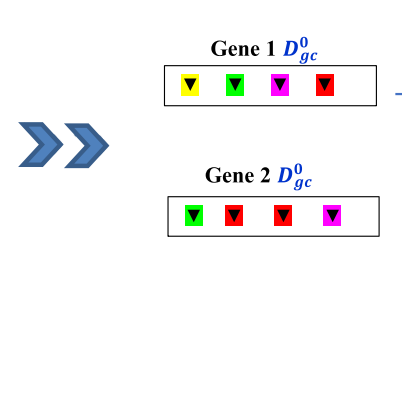

D. Compute the Sum of Most deleterious score in each individual gene across all patients into $D_{g c}^{m}$

Gene 1; $1000 D_{g c}^{m}$

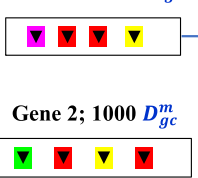

Fig. 1 SMDS, a gene-based permutation method for the detection of candidate driver genes. The steps are shown from a to $\mathbf{e}$

$<0.7)$ and the remaining 13 pairs have a lower correlation $(r \leq 0.4)$ for breast and lung cancer. Additional file 1: Figure S1 presents UPGMA (Unweighted Pair Group Method with Arithmetic Mean) dendrograms clustering of scores according to their pairwise scores distance between scores measured by $1-r$.

\section{Analysis of Most deleterious scores and sum of Most deleterious scores}

We next identified the $\operatorname{MDS}\left(\boldsymbol{X}_{\boldsymbol{g s c}}\right)$ of each score in each gene for each sample. We also computed the SMDS ( $D_{g c}^{\boldsymbol{o}}$ ) for each gene across all samples. Our analysis included 19,835 protein coding genes in breast cancer and 20,047 protein coding genes in lung cancer after the annotation process. For four pan-genome mutation predictive tools (CADD, DANN, Fathmm-MKL coding and Fathmm-MKL noncoding), each gene has a SMDS $\boldsymbol{D}_{\boldsymbol{g} \boldsymbol{c}}^{\boldsymbol{o}}>$ 0.0 ; meaning that at least one observed SNV in those genes was scored. However, for the three missense and splicing mutation scoring methods (MetaLR, SPIDEX and VEST3), because a higher proportion of SNVs are not missense or splicing mutations there are multiple genes with SMDS $\boldsymbol{D}_{g c}^{\boldsymbol{o}}=0.0$. For instance, only 17 to $22 \%$ and 46 to $49 \%$ of the genes have $D_{g c}^{o}>0.0$ for missense scores (MetaLR and VEST3) and SPIDEX, respectively (Additional file 1: Figure S2).

\section{Finding candidate driver genes}

We applied our gene-based permutation model to 19,835 and 20,047 protein coding genes identified from the annotation process (see Methods) for the breast and lung cancer data, respectively. We define candidate driver genes as those with $p$-values less than or equal to 0.01 (p-value $\leq 0.01$ ). We found that a long list of genes met that criteria including 942 (4.7\%) unique genes for breast and 796 (4.0\%) for lung cancer (Table 1). Depending on the individual predictive method used in the permutation model: 0.8 to $1.3 \%$ genes showed statistically significant results for breast cancer and 0.6 to $0.9 \%$ genes for lung cancer. Additional file 1: Figure S3 presents the null distribution of TP53 ( $p$-value $<0.01$ ), a known breast cancer gene, and SLC1A2 ( $p$-value $=$ 0.195), a non-cancer gene, for the CADD score.

\section{$P$-value distributions of the seven permutation models}

The performance of the $p$-value distribution of the seven permutation models were evaluated using quantilequantile (QQ) plots, which displays the relationship between the observed $p$-values to the expected uniform distribution of $p$-values under the null hypothesis. For 
Table 1 Candidate driver genes positively selected ( $p$-value $\leq 0.01$ ) by each permutation model and their percentage (in brackets) of all genes tested for breast and lung cancer data

\begin{tabular}{|c|c|c|c|c|c|c|c|c|c|}
\hline Permutation model & CADD & DANN & Fathmm-MKL coding & Fathmm-MKL noncoding & MetaLR & SPIDEX & VEST3 & Unique genes & Total genes \\
\hline Breast & $263(1.3)$ & $158(0.8)$ & $178(0.9)$ & $184(0.9)$ & $174(0.9)$ & $178(0.9)$ & $171(0.9)$ & $942(4.7)$ & 19,835 \\
\hline Lung & $121(0.6)$ & $142(0.7)$ & $138(0.7)$ & $149(0.7)$ & $178(0.9)$ & $171(0.9)$ & $164(0.8)$ & $796(4.0)$ & 20,047 \\
\hline
\end{tabular}

breast cancer (Fig. 2a), most QQ plots for the individual permutation models show that the majority of genes fit the null expectations and only a small proportion of genes having a smaller $\mathrm{p}$-value than expected. Indeed, the deviation of $p$-values from the expected distribution of $p$-values; as observed outside the $95 \%$ confidence interval (grey shading) at the top tail suggest candidate genes. Individual QQ plot for each independent model are shown in Additional file 1: Figure S4. The same trend was observed for the p-values for lung cancer genes (Fig. 2b and Additional file 1: Figure S5).

\section{Agreement in predicting candidate driver genes by the seven independent permutation models}

To compare the predictions of the seven permutation models, we collected 942 unique genes positively selected ( $p$-values $\leq 0.01$ ) by each model from breast cancer and 796 genes for lung cancer patients. We started by assessing the agreement between all the seven models for breast cancer (Additional file 2: Table S3) and lung cancer (Additional file 2: Table S4). We identified the proportion of selected genes that were unique to each predictor or commonly chosen by two to three, or by more than three other permutation models for breast cancer (Fig. 3a) and lung cancer (Fig. 3b). For the pan-genome scores and across the two cancer tissues types, more agreement can be seen among CADD, DANN, Fathmm-MKL coding, and Fathmm-MKL noncoding compared to the three predictors scoring only coding or splice regions. For instance, in breast cancer, we found that taken individually each score selected roughly half of the genes $(55,43,42$ and $51 \%)$ unique to its own; whilst the remaining half were also selected by other scores. On the other hand, VEST3 has less unique genes (38\%) compared to other predictors; MetaLR was comparable to the pan-genome scores (50\%), whereas for SPIDEX most of its selected genes (88\%) were unique to its own. In lung cancer (Fig. 3b) the same trend is observed among pan-genome scores that selected roughly one third to half of the genes unique to its own; whilst the remaining half to two third were also selected by other scores. VEST3 and MetaLR scores have unique genes comparable to the pan-genome scores; whereas for SPIDEX the vast majority of genes selected genes $(89 \%)$ were unique to their own respectively breast and lung cancer.
Figure 4 presents a matrix display of the intersection of the number of the breast candidate driver genes selected by the seven scores. Set intersections characterize common genes predicted by a set of scores. The blue circle in the matrix label scores that are part of the intersection. The results show that for breast cancer more genes were exclusively selected by only one score demonstrating the divergence of these methods: SPIDEX (157), CADD (141), Fathmm-MKL noncoding (93), MetaLR (87), Fathmm-MKL coding (75), DANN (67), and VEST3 (65). Moreover, fewer candidate genes were selected by a set of two scores CADD and DANN (60), MetaLR and VEST3 (46), Fathmm-MKL coding and Fathmm-MKL noncoding (37). The same trend is observed as the number of consensus scores increased till seven. We noted that intersecting the scores resulted in very few overlapping protein coding genes. There was one common gene (TP53) selected by all seven scores; 2 genes (GRIN1, XG) by six scores; 6 genes (TAF1L, MAP 3 K1, PIK3CA, OTOP1, PSMA4, FZD3) by five scores; and 13 genes by four scores (KMT2C, RTDR1, MICAL2, CBFB, SHBG, CDH10, C9orf135, GABRR1, ODAM, PHTF2, GANC, MAP 2 K4, FUNDC2) and 50 genes by three scores (Fig. 4a and Additional file 2: Table S3).

For lung cancer, there was one common gene (DLX4) selected by six scores; 4 genes (TP53, CCT7, ST6GAL2 and RBM10) by five scores; 16 genes (LY6G6E, STK11, MUSTN1, NF1, FBXW7, OR7C1, SLC27A1, SIRPD, CTIF, CEP250, LPA, RYR1, QRSL1, CHD3, KCNMB3) by four scores and 27 genes by three scores (Fig. $4 \mathrm{~b}$ and Additional file 2: Table S4).

Next, we considered the agreement between the four models with pan-genome scores (CADD, DANN, Fathmm-MKL coding, and Fathmm-MKL noncoding). For breast cancer, these four scores were able to predict 583 unique genes (62\%) out of the total 942 candidate genes. DANN detected fewer driver candidates (158) and CADD detected the highest number (263) (Table 1). Overall, 7 genes were commonly predicted by the four models (Additional file 1: Figures S6A and S7A). More genes were exclusively predicted by only one predictive model demonstrating the divergence of these models: CADD (156), Fathmm-MKL noncoding (100), Fathmm-MKL coding (97), and DANN (69). We found there was more agreement between some of these scores. We observed a higher number of genes selected by a combination of CADD and 

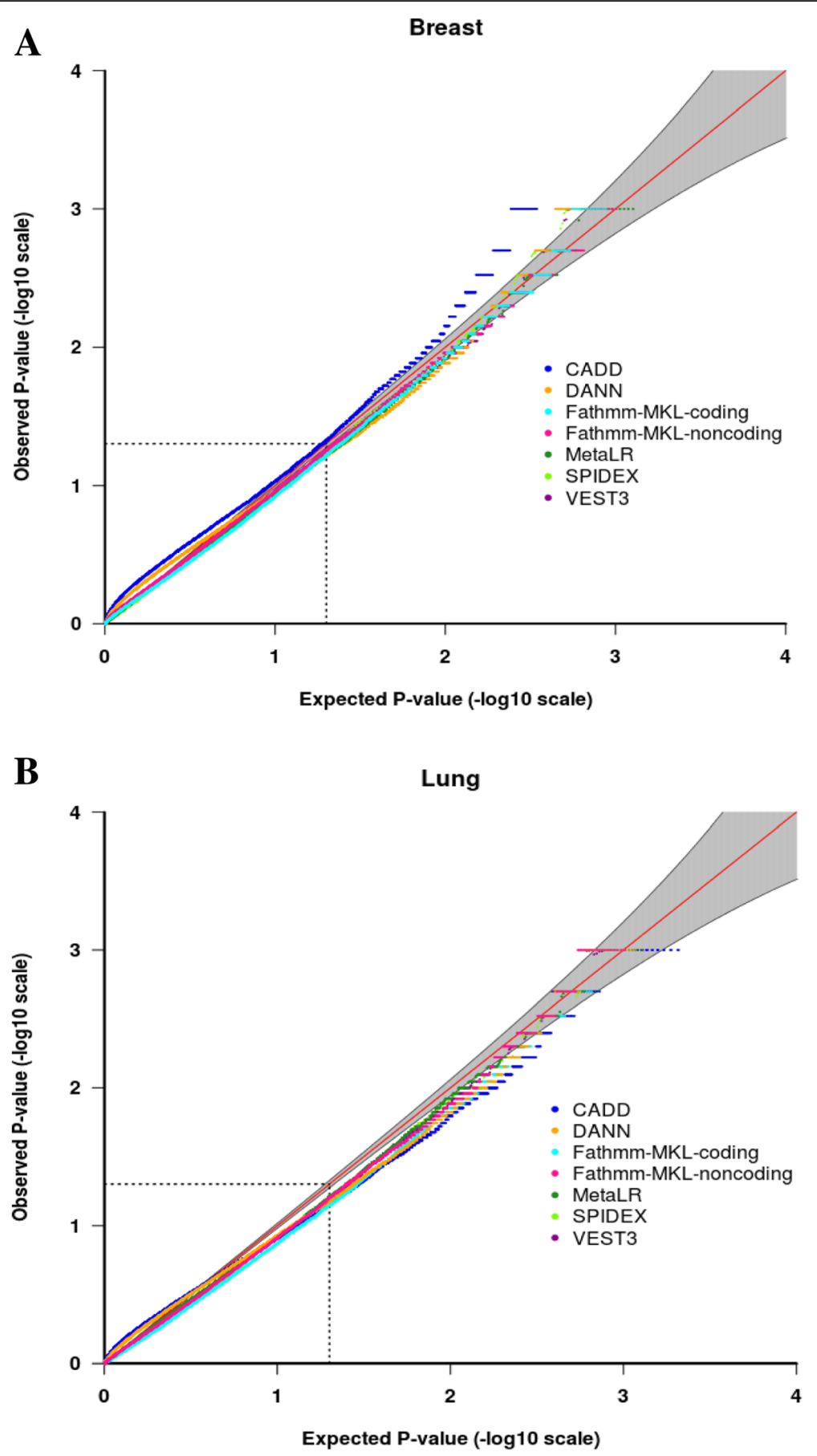

Fig. 2 Quantile-quantile (QQ) plots of $p$-values comparing the observed distribution of $\mathrm{p}$-values ( $\mathrm{y}$ - axis) to the expected $p$-values of a null distribution ( $x$ - axis) for 19,835 breast cancer genes (Panel a) and 20,047 lung cancer genes (Panel b). The red line represents the expectation under the null. The grey area depicts the $95 \%$ confidence

DANN (65), and Fathmm-MKL coding and Fathmm-MKL noncoding (48) and that was also noted in lung cancer. This higher correlation is expected as CADD and DANN shared the same training data, as well as Fathmm-MKL coding and noncoding.
We also assessed the agreement between the remaining three models for breast cancer. Two of these independent models only score missense mutations in coding regions (VEST3 and MetaLR) whereas SPIDEX cover exonic and intronic regions near splicing sites. 

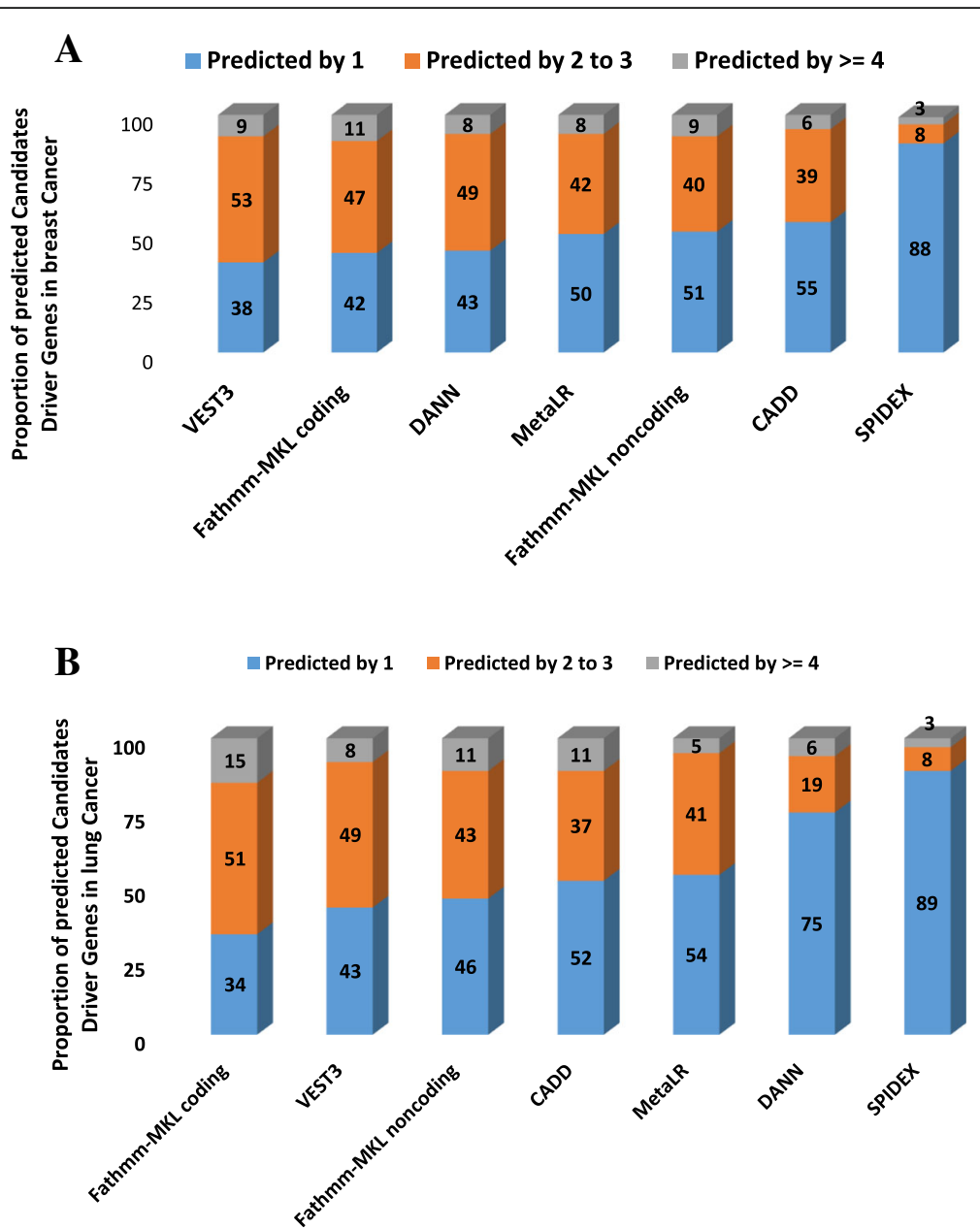

Fig. 3 Proportion of breast cancer candidate driver genes (Panel a) and lung cancer genes (Panel b) predicted by one, two to three or more than three permutation models

Across the two cancer tissue types, they have provided the MDS ( $\boldsymbol{D}_{g c}^{\boldsymbol{o}}$ ) for only 17 to $49 \%$ of the genome (Additional file 1: Figure S2). We found that for breast cancer, these three predictors selected 437 unique cancer candidate driver genes $(46 \%)$ out of the 942 candidate driver genes. They have consensus on 5 genes (TP53, RAB37, ODAM, FZD3 and FUNDC2) (Additional file 1: Figures $\mathrm{S} 6 \mathrm{~B}$ and $\mathrm{S7B}$ ). More genes were exclusively selected by only one predictor demonstrating the divergence of these methods: SPIDEX (167), MetaLR (99), and VEST3 (90) (Additional file 1: Figure S3B). We observed many shared predicted genes (70) between MetaLR and VEST3 (Additional file 1: Figure S7B).

For lung cancer, the four models with pan-genome scores (CADD, DANN, Fathmm-MKL coding, and Fathmm-MKL noncoding) selected 419 unique genes (53\%) out of the total 796 candidate genes. As shown in Additional file 1: Figure S8A and S9A, they commonly predicted 7 genes (LY6G6E, TP53, STK11, MUSTN1, RBM10, DLX4 and CCT7). The remaining three models
(MetaLR, VEST3 and SPIDEX) selected 435 unique cancer candidate driver genes (55\%) out of the 796 candidate driver genes. Additional file 1: Figures S8B and S9B show that they have consensus on 5 genes (KCNC3, SPINK14, KCNMB3, CHD3, and ST6GAL2).

\section{Intersection of the predicted candidate driver genes for breast and lung cancer with the Cancer genes census} The CGC currently lists 534 genes (with somatic mutations) causally associated with different types of cancers. Among them 32 genes have been implicated in breast cancer. If considering only genes predicted by five or more scores, the seven predictive permutation models selected a total of 10 protein coding genes for breast cancer (Table 2). Among them, TP53 was selected by all seven scores and is known to cause breast cancer. Two other genes, PIK3CA and MAP $3 \mathrm{~K} 1$, which were selected by five scores, are also associated with breast cancer according to CGC. MAP $2 \mathrm{~K} 4$ were also selected by five scores and is a known to be associated with other 


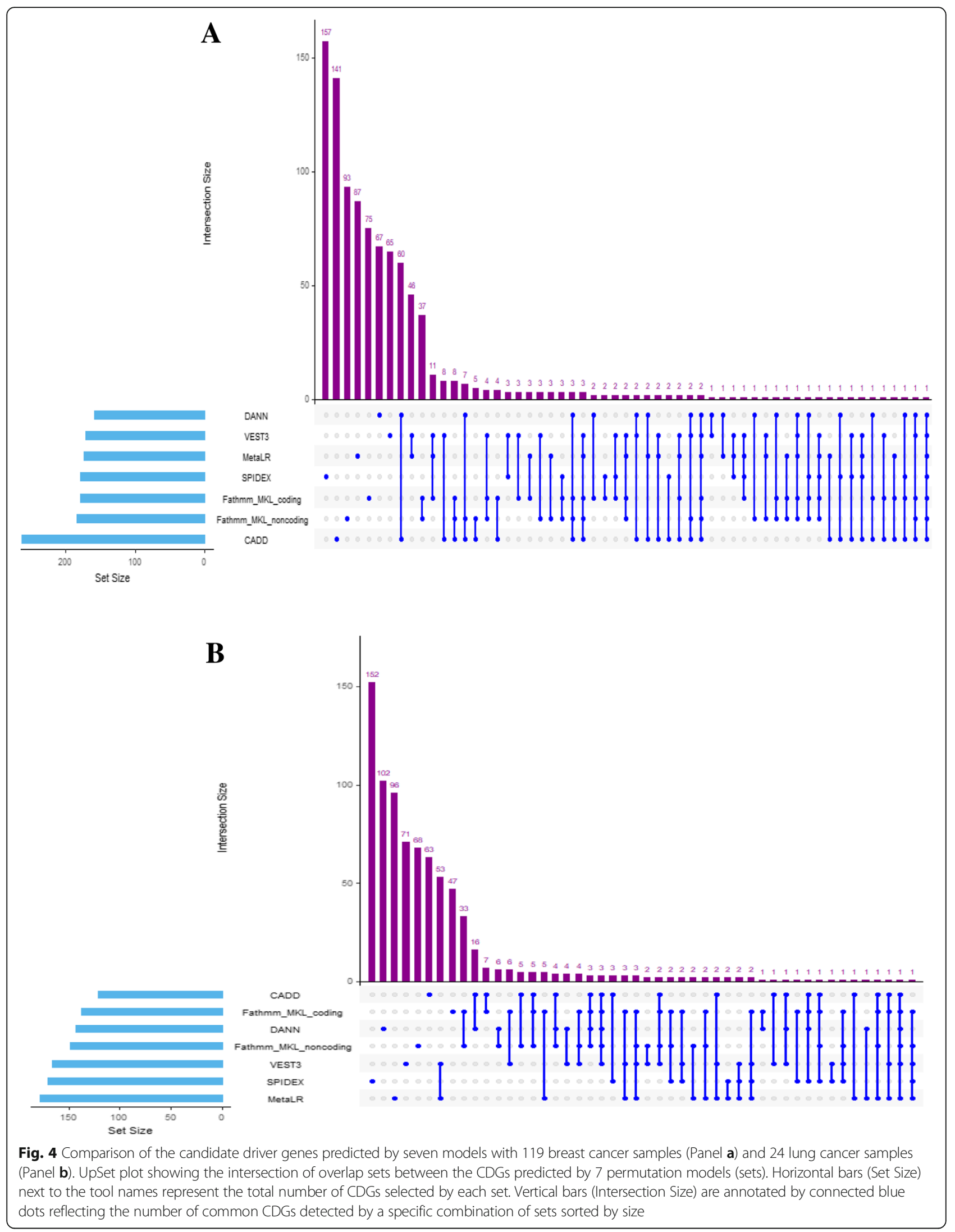


Table 2 Candidate driver genes predicted by five or more permutation models for breast and lung cancer

\begin{tabular}{|c|c|c|c|c|}
\hline $\begin{array}{l}\text { Number of shared } \\
\text { predictive models }\end{array}$ & $\begin{array}{l}\text { Number of genes } \\
\text { predicted }\end{array}$ & $\begin{array}{l}\text { Genes names overlapping with } \\
\text { breast or lung cancer genes in CGC }\end{array}$ & $\begin{array}{l}\text { Genes names overlapping with } \\
\text { other cancer genes in CGC }\end{array}$ & Gene names not in CGC \\
\hline \multicolumn{5}{|l|}{ Breast } \\
\hline 7 & 1 & TP53 & & \\
\hline 6 & 2 & & & GRIN1, XG \\
\hline 5 & 6 & PIK3CA, MAP $3 \mathrm{~K} 1$ & MAP 2 K4 & TAF1L, OTOP1, PSMA4, FZD3 \\
\hline \multicolumn{5}{|l|}{ Lung } \\
\hline \multicolumn{5}{|l|}{7} \\
\hline 6 & 1 & & & DLX4 \\
\hline 5 & 4 & TP53, RBM10 & & CCT7, ST6GAL2 \\
\hline
\end{tabular}

cancer types. The remaining six genes (GRIN1, XG, TAF1L, OTOP1, PSMA4, and FZD3) are not listed in CGC. Additional file 1: Table S5 shows that all together these models were able to predict 15 (47\%) of known breast cancer genes. Additional file 1: Table S6 lists the $p$-values of 32 breast cancer genes where 38, 25, 19, 19, 19,16 , and $13 \%$ of CGC genes were selected by CADD, VEST3, Fathmm-MKL coding, MetaLR, SPIDEX, Fathmm-MKL noncoding and DANN permutation models respectively. We also found that a total of 37 candidates predicted by the seven permutation models for breast cancer have already been linked to other types of cancer (Additional file 1: Table S5). Figure 5a shows the fraction of the 534 genes in CGC predicted by each model.

In CGC, twelve genes with somatic mutations are causally associated with lung cancer. A total of 5 protein coding genes were predicted by five or more scores in this study. Among them, TP53 and RBM10 were selected by five scores are known to cause lung cancer according to CGC. The remaining three genes (DLX4, CCT7, and ST6GAL2) are not listed in CGC. Additional file 1: Table S7 shows that all together the seven models were able to predict $2(6 \%)$ of known lung cancer genes. Additional file 1: Table S8 lists the $p$-values of 12 lung cancer genes found in CGC. Figure 5b shows the fraction of the 534 genes in CGC predicted by each model. We found that a total of 22 candidates predicted by the seven permutation models for lung cancer have already been linked to other types of cancer (Additional file 1: Table S7).

\section{Overall performance of the seven deleteriousness prediction scores}

The overall performance of the seven permutation models was evaluated using the following three criteria including the number of genes predicted by each model, overlap with the CGC and the model consensus. These criteria were recently recommended by Tokheim et al. [13] for assessing the performance of driver gene prediction method in absence of a gold-standard method. The model consensus is the fraction of predicted candidate driver genes selected by two or more other predictors (Fig. 3a and b). The overlap with the CGC represent the fraction of the 534 genes in CGC predicted by each model (Fig. 5a and b). In this study, the top ranked scores for breast cancer are VEST3, Fathmm-MKL coding and CADD. For lung cancer, Fathmm-MKL coding, CADD, and VEST3 outperformed the other (Table 3).

\section{Discussion}

In this study we developed a function prediction based approach (SMDS) utilizing both coding and noncoding deleteriousness prediction scores for somatic SNVs observed in whole genome sequence data of cancer samples to identify potential cancer driver genes. We applied this approach to breast and lung cancer data sets using seven different functional prediction scores (CADD, DANN, Fathmm-MKL coding, Fathmm-MKL noncoding, MetaLR, SPIDEX and VEST3). A total of 942 unique gene were selected by the seven scores with $p$-values $\leq 0.01$ in our permutation tests for breast cancer. Among them, ten protein coding genes were selected by five or more scores, which have higher likelihood to be true cancer driver genes. Among the ten genes, the well-known cancer driver gene TP53 is the only gene selected by all seven scores. PIK3CA, MAP 3 $\mathrm{K} 1$ and MAP $2 \mathrm{~K} 4$ are selected by five scores and known to be associated with breast cancer or other cancer types. Six new candidate genes were identified by at least five of the seven scores but not listed in the CGC: GRIN1, XG, TAF1L, OTOP1, PSMA4 and FZD3. However, there are some evidence supporting their involvement in cancer development.

For lung cancer, a total of 796 unique gene were selected by the seven scores with $\mathrm{p}$-values $\leq 0.01$ in our permutation tests. Among them, five protein coding genes were selected by five or more scores. Two are known lung cancer genes (RBM10 and TP53). The fewer number of know cancer genes predicted here for lung cancer may be attributed to the small sample size (24 

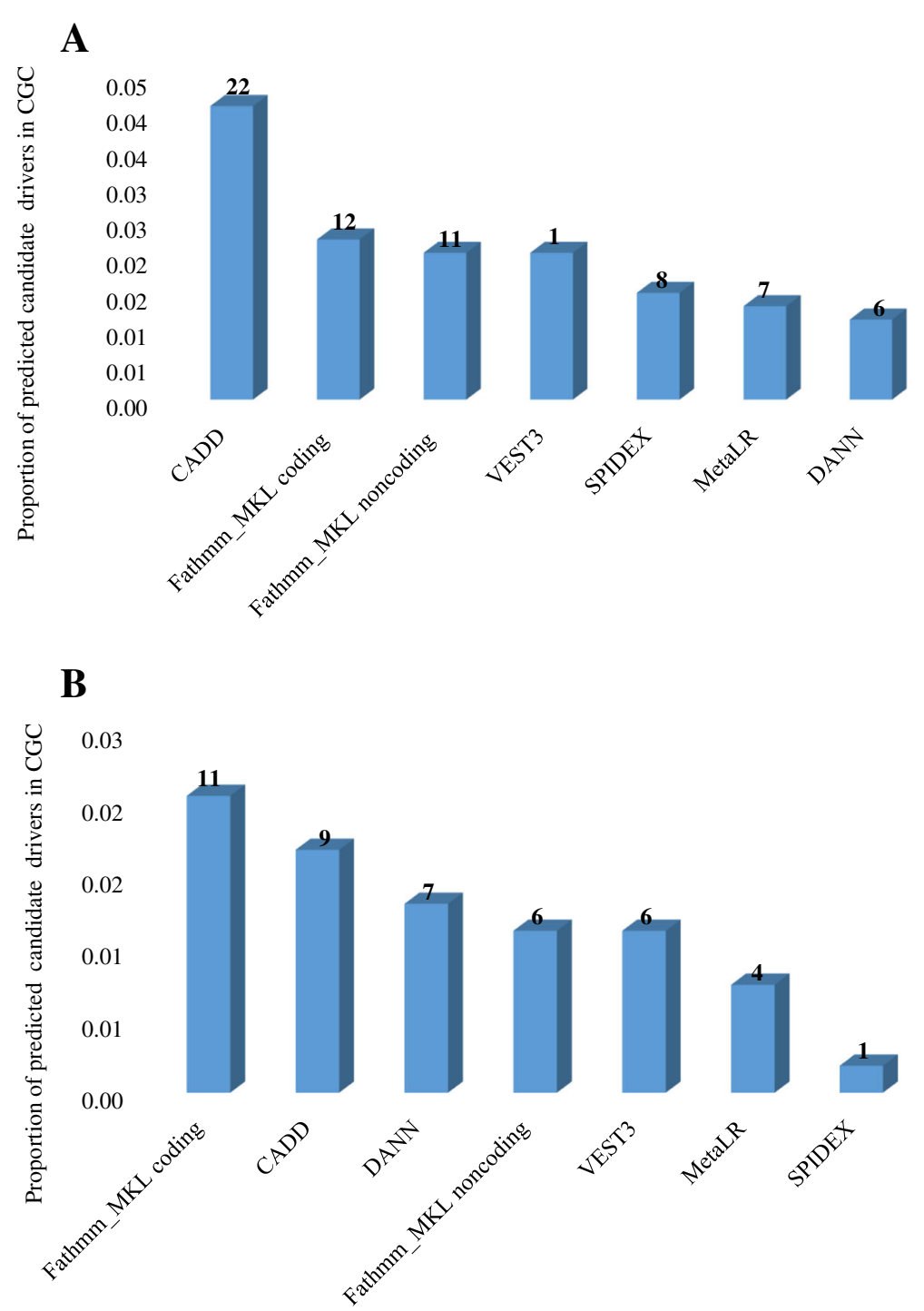

Fig. 5 Proportion of 534 genes in the Cancer Gene Census predicted by each permutation model from breast cancer (Panel a) and lung cancer (Panel b). The number of predicted driver genes is on top of each bar

samples compared to 119 samples in breast cancer) and higher level of passenger mutations (468,348 SNVs for lung cancer samples compared to 278,152 SNVs for breast cancer samples). Three new candidate genes identified by at least five of the seven scores are not listed in the CGC (DLX4, CCT7 and ST6GAL2). There are also evidence supporting their involvement in cancer development.

\section{PSMA4}

The results of a recent study showed that mRNA high expression level of PSMA4 in multiple cancer types were significantly associated with worse prognostic in breast cancer, gastric cancer and HER2-negative gastric cancer; whereas they were correlated with better prognostic in lung adenocarcinoma [14]. A previous functional study reported the gene was involved in promoting cancer cell proliferation and apoptosis; and it was labeled as a "strong candidate mediator" associated with lung cancer susceptibility [15]. PSMA4 polymorphism has been associated to lung cancer risk in Chinese Han population [16]. The gene was also overexpressed in colorectal cancer patients and was significantly correlated with metastasis development and worse prognosis [17].

\section{TAF1L}

This gene was previously reported as the fourth significantly mutated gene among 20 protein kinase genes rated by probability of harboring at least one mutation [18]. It was later identified as a potential driver gene with clinical relevance in melanoma cancer samples [19]. Interestingly, the authors found that it was recurrently 
Table 3 Performance comparison of the seven permutation models on breast and lung cancer

\begin{tabular}{|c|c|c|c|c|c|c|}
\hline Method & Number of Significant genes & Overlap with CGC & Method consensus & CGC Rank & Consensus Rank & Average Rank \\
\hline \multicolumn{7}{|l|}{ Breast cancer } \\
\hline$C A D D$ & 263 & 0.041 & 0.45 & 1 & 4 & 2.5 \\
\hline DANN & 158 & 0.011 & 0.57 & 6 & 2 & 4 \\
\hline Fathmm-MKL coding & 178 & 0.022 & 0.58 & 2 & 2 & 2 \\
\hline Fathmm-MKL noncoding & 184 & 0.021 & 0.49 & 3 & 3 & 3 \\
\hline MetalR & 174 & 0.013 & 0.5 & 5 & 3 & 4 \\
\hline SPIDEX & 178 & 0.015 & 0.12 & 4 & 5 & 4.5 \\
\hline VEST3 & 171 & 0.021 & 0.62 & 3 & 1 & 2 \\
\hline \multicolumn{7}{|l|}{ Lung cancer } \\
\hline$C A D D$ & 121 & 0.017 & 0.48 & 2 & 4 & 3 \\
\hline DANN & 142 & 0.013 & 0.25 & 3 & 6 & 4.5 \\
\hline Fathmm-MKL coding & 138 & 0.021 & 0.66 & 1 & 1 & 1 \\
\hline Fathmm-MKL noncoding & 149 & 0.011 & 0.54 & 4 & 3 & 3.5 \\
\hline MetaLR & 178 & 0.007 & 0.46 & 5 & 5 & 5 \\
\hline SPIDEX & 171 & 0.002 & 0.11 & 6 & 7 & 6.5 \\
\hline VEST3 & 164 & 0.011 & 0.57 & 4 & 2 & 3 \\
\hline
\end{tabular}

mutated in pan-negative melanoma samples (without mutations in known melanoma cancer driver genes). The gene was found to be disrupted by frameshift mutations in Gastric and Colorectal Cancers [20] and because of its known involvement in apoptosis induction and cell cycle regulation; they hypothesized that the presence of frameshift mutations could decrease the cell death and therefore lead to higher survival of cancer cells in gastric and collateral cancer patients.

\section{GRIN1}

Recent studies have demonstrated the role GRIN1 plays in tumorigenesis. One study [21] analyzed expression of GRIN1 in 12 different human tumor cell lines and concluded it was present in 9 of them including breast cancer. The gene is a calcium regulating tumor suppressor that was reported as one of the six hyper mutated genes impacting dysregulation of the glutamate signaling pathways in melanoma [22]. Functional mutations (loss of function) in this gene have been linked to tumor growth, proliferation and survival in melanoma. Another study [23] reported that functional receptors from this gene were crucial for maintaining tumor cell growth and viability in breast and could by target for the development of therapeutic drugs.

\section{FZD3}

This gene was previously described as an oncogene and a probable therapeutic target gene [24]. The gene was found to be overexpressed in multiple cancer types including lung, leukemia, myeloma, lymphoma and sarcoma. A study assessing its clinical significance in colorectal cancer concluded that FZD3 was not only associated with carcinogenesis and progression; but also, its staining could be used as prognostic marker [25].

\section{OTOP1}

This gene has been associated to diverse type of cancer including esophageal adenocarcinoma, pancreas, Melanoma, Lung, and prostate [26]. It was frequently mutated in lung cancer cell line genomes [27] and pancreatic tumors [28], but it was not conclusively classified as a driver gene.

\section{XG}

This gene is known to be associated to lower survival and tumor invasiveness in Ewing's Sarcoma (EWS) patients and was described as a biological marker for EWS [29, 30].

\section{DLX4}

The methylation of DLX4 was strongly associated with high risk of recurrence and poor prognostic survival in lung cancer patients [31]. The gene can drive tumor progression in ovarian cancer through the NF- $\mathrm{kB}$ pathway by activating a regulatory factor cell surface molecule CD44 [32]. It was also found to promote ovarian cancer by inducing the expression of iNOS, an enzyme that stimulates angiogenesis [33].

\section{ST6GAL2}

DNA methylation of ST6GAL2 has been proposed as a cancer biomarker for screening and detection. A recent study had linked ST6GAL2 hypermethylation to cervical 
intraepithelial neoplasia grade 3 or worse [34]. ST6GAL2 upregulation may be implicated to growth and proliferation in invasive ductal carcinoma (IDC) [35]. In follicular thyroid carcinoma, up-regulated ST6GAL2 in advanced cells and its co-expression with LncRNA HCP5 was strongly associated to cell proliferation, migration, invasiveness and angiogenesis [36].

\section{CCT7}

This gene has been recently identified as a potential biomarker for endometrial carcinoma [37]; the gene was found to be highly expressed in a proteomic analysis comparing endometrial carcinoma and normal precarious tissues. According to the same authors, CCT7 has been linked to multiples cancer (neck cancer, adenocarcinoma, carcinoma squamous cell, neoplasms, malignant neoplasms and lymphoma) and health conditions (necrosis, staphylococcal scalded skin syndrome and Hodgkin disease). CCT7 was also identified as a biomarker linked to late stage colorectal cancer in a protein interaction sub-networks analysis for early tumorigenesis comparing normal and late stage colon cancer tissues [38]. A study comparing levels of mRNA expression during overall survival in glioblastoma multiform (GBM) human patients and protein expression during development of the macaque rhesus brain discovered eight signature genes including CCT7 that were higher expressed in early brain development, were associated with overall survival of in GBM patients and have the potential for drug target therapy [39].

\section{Conclusion}

In this paper we discussed a gene-based permutation approach (SMDS) that functionally interrogates the whole genomes of cancer patients to identify potential candidate driver genes. We have performed a comprehensive analysis to predict CDGs by applying the SMDS method to breast and lung cancer data and comparing the scores of seven popular functional predictive methods. Each individual SMDS was able to identify a set of potential CDGs. We intersected CDGs predicted by at least five of the seven SMDS models and obtained a list of well-known cancer genes reported in the CGC and also novel CDGs that are worth further investigation. Our study also showed the advantages of utilizing pan-genome deleteriousness prediction scores in function prediction based methods for identifying cancer driver genes. Although for breast cancer the best performed score is tied between a missense prediction score, VEST3, and a pan-genome score, Fathmm-MKL coding, for lung cancer, on the other hand, two pan-genome scores, CADD and Fathmm-MKL coding performed better than missense prediction scores. Considering the pan-genomes scores' performances are at least comparable to missense prediction scores yet provide complementary information, they shall be included in function prediction based approaches for detecting CDGs, as demonstrated in this study.

\section{Methods \\ Cancer mutation data collection}

We curated somatic SNVs primary from the whole genome sequence data of two cancer tissue types $(278,152$ SNVs from 119 breast cancer patients and 468,348 SNVs from 24 lung cancer patients) of a large published dataset containing both whole-genome sequencing and whole-exome sequencing data [6]. These somatic mutation data were obtained as follows as described by the authors. Normal DNA samples and tumor samples of the same individual were sequenced. All somatic mutations data of each sample was then combined to generate its mutational catalog.

\section{Individual predictive methods}

The seven individual scores included in our analysis were initially developed to prioritize functional mutations and all are non-cancer specific (Additional file 1: Table S1). CADD [7], is a meta-annotation tool that contrasts existing genomic variation to simulated genomic variation. It uses a linear kernel SVM to differentiate benign variants from deleterious variants (binary classification) by integrating the information of diverse functional annotations (evolutionary conservation, regulatory and transcript information, and protein-level scores) into a single score. It scores the deleteriousness of SNVs as well as insertion/deletions variants for both coding and non-coding regions. DANN [8] algorithm uses the same features set and training data as CADD to train a DNN which scores every possible SNVs in order to capture non-linear relationships among features. Fathmm-MKL [9] predicts the functional, molecular and phenotypic consequences of SNVs of both coding and noncoding regions. It uses a MKL classifier to combine ten different features groups including functional annotations from ENCODE and nucleotide-based conservation measures. Two scores were produced by this method based different training features: coding and noncoding. Both scores are pan-genome. MetaLR [10] scores the deleteriousness of missense SNVs. It combines individual scores from ten predictors including nine scores (SIFT [40], PolyPhen-2 [5], GERP++ [41], MutationTaster [42], Mutation Assessor [43], Fathmm [44], LRT [45], SiPhy [46], PhyloP [47]) and the maximum minimum frequency observed in the 1000 genomes populations into one ensemble score using a LR model. SPIDEX [11] algorithm is a Bayesian ensemble of DNN trained with RNA sequencing data. It scores all synonymous, missense and nonsense exonic SNVs, as well as intronic SNVs that are up to $300 \mathrm{nt}$ from splice junctions. VEST3 [12] method 
integrated 86 features from SNVBox [48] (conservation scores, amino acid residue substitution scores, UniProtKB annotations, and predicted local protein structure) to predict the functional significance of missense mutations.

\section{Annotation and scoring of variants}

SNV annotation was done through the WGSA pipeline [49]. Three different annotation software (ANNOVAR [50], SnpEff [51] and VEP [52]) with two different databases (RefSeq [53] and Ensembl [54]) were used to functionally annotate all the SNVs. After the annotation process, we retrieve the following four VEP gene annotation fields: VEP_ensembl_Consequence, VEP_ensembl_Gene_Name, VEP_ensembl_Protein_ID, and VEP_ensembl_CANONICAL. We filter only protein coding genes from other genes using the VEP_ensembl_Protein_ID. Because some genes have more than one transcript, we restricted our analysis to the canonical transcripts. The region of each gene was defined as from $5 \mathrm{~kb}$ upstream to $5 \mathrm{~kb}$ downstream. All seven scores (CADD, DANN, Fathmm-MKL noncoding and Fathmm-MKL coding, VEST3 and MetaLR) for each SNV were annotated through WGSA [49]. To make the individual scores comparable to one another CADD and SPIDEX scores were rescaled to rank between 0 and 1 . Because not all the seven scoring systems are applicable to the entire genome; three methods including MetaLR and VEST3 (score only missense SNVs) and SPIDEX (score only part of exon and intron regions) had a high number of variants with missing values. The lowest score (0.0) was used for those variants.

\section{A gene-based permutation model}

We designed a gene-based permutation model to prioritize cancer candidate driver genes in light of the InVeX method [4]. We applied this new approach to compare the performance of seven predictive methods (CADD, DANN, Fathmm-MKL noncoding and Fathmm-MKL coding, MetaLR, SPIDEX and VEST3) that measure the functional effect of SNVs in both coding and non-coding regions of the genome. For a cancer tissue type and assuming there are total of $(G)$ genes, $(S)$ samples, and $(C)$ categories (predictive scores of each individual predictive method); first, we identify the MDS $\left(X_{g s c}\right)$ of each observed SNV, for each sample $(s)$, gene $(g)$ and category/score $(c)$. MDS is a monochromic measure of deleteriousness ranging from 0 to 1 ; and the larger the score, the more likely the variant is deleterious). Second, we compute the SMDS where all the MDS per sample $(s)$ are tallied for each gene $(g)$ and each category (c) into $D_{g c}^{o}=\sum_{s=1}^{S} X_{g s c}$. Third, we generate within-gene null distribution to compute $p$-values in the observed data. Basically, for each sample $(s)$, gene $(g)$ and category $(c)$, the gene-based permutation approach randomly permuted the position of the each observed SNV along the gene sequence, preserving the trinucleotide context in each sample (i.e. for a ACG > ATG SNV we randomly choose another ACG trinucleotide sites from the gene sequence, from $5 \mathrm{~kb}$ stream to $5 \mathrm{~kb}$ downstream, and "move" the ACG > ATG SNV there) and this is done 1000 times. Next, the newly "simulated" SNVs were annotated and scored and each set is known as a trial (total number of trials $=1000)$. Fourth, for each trial set, we identify the MDS $\left(X_{g c s}^{m}\right)$ and compute the SMDS $\left(D_{g c}^{m}\right.$ $\left.=\sum_{s=1}^{S} X_{g s c}\right)$ for the simulated data. Fifth, we compute individual p-value $\left(P_{g c}^{o}\right)$ for each gene $(g)$ and for each category $(c)$ for the observed $D_{g c}^{o}$ based on the empirical null distribution of the simulated $1000 D_{g c}^{m}$ scores $(\mathrm{m}=$ 1000). $P_{g c}^{o}$ was defined as the percentage of the simulated $D_{g c}^{m}$ scores equal or greater than the observed $D_{g c}^{o}$.

\section{Plotting}

The QQ-plot of p-values showing the distribution of $\mathrm{p}$-values were produced in $\mathrm{R}$ using the function $\mathrm{pQQ}$ from the Haplin library [55]. The intersection between the candidate driver genes predicted by each individual predictive model were found using UpSetR [56]. The Veen diagrams were drawn in the website http://bioinformatics.psb.ugent.be/webtools/Venn/. The dendrogram (UPGMA cluster analysis) were produced in $\mathrm{R}$ using the package UPGMA, (https://www.rdocumentation.org/packages/phangorn/versions/2.4.0/topics/ upgma).

\section{Additional files}

Additional file 1: Figure S1. UPGMA dendrogram comparing the seven prediction scores for breast cancer (Panel A) and lung cancer (Panel B). Figure S2. Percentage of genes with Sum of Most Deleterious Scores $D_{g c}^{\circ}$ $>0.0$ covered by each of the 7 predictive models in for breast and lung cancer data. Figure S3. Null distribution of the permuted Sum of Most Deleterious Scores $D_{g c}^{m}$ for the CADD score in TP53 ( $p$-value $\left.=0.000\right)$ well-known breast cancer gene; and SLC1A2 ( $p$-value $=0.195)$ gene not associated with cancer. The red dots and lines indicate the observed values 6.7 for TP53 and 3.7 for SALL4. Figure S4. Quantile-quantile plot of the observed $p$-values for breast cancer genes ( $y$ - axis) against the expected $P$ values of a null distribution ( $x$ - axis). The red line represents the expectation under the null hypothesis. The grey area depicts the $95 \%$ confidence interval. Figure S5. Quantile-quantile plot of the observed p-values for lung cancer genes ( $y$ - axis) against the expected $P$ values of a null distribution ( $x$ - axis). The red line represents the expectation under the null hypothesis. The grey area depicts the 95\% confidence interval. Figure S6. Proportion of breast candidate driver genes predicted by one, two to three, and more than three permutation models: Panel AAgreement between CADD, DANN, Fathmm-MKL coding and FathmmMKL noncoding; Panel B- Agreement between MetaLR, SPIDEX and VEST3. Figure S7. Comparison of breast candidate genes driver predicted by seven independent permutation models. Panel A- Venn diagram of candidate driver genes predicted by CADD, DANN, Fathmm-MKL coding, and Fathmm-MKL noncoding. Panel B- Venn diagram of candidate driver 
genes predicted MetaLR, SPIDEX and VEST3. Figure S8. Proportion of lung candidate driver genes predicted by one, two to three, and more than three permutation models: Panel A- Agreement between CADD, DANN, Fathmm-MKL coding and Fathmm-MKL noncoding; Panel BAgreement between MetaLR, SPIDEX and VEST3. Figure S9. Comparison of lung candidate driver genes predicted by seven independent permutation models. Panel A- Venn diagram of candidate driver genes predicted by CADD, DANN, Fathmm-MKL coding, and Fathmm-MKL noncoding. Panel B- Venn diagram of candidate driver genes predicted MetaLR, SPIDEX and VEST3. Table S1. Summary of methods for scoring somatic mutations (SNVs) deleteriousness. Table S2. Pearson's correlation Coefficients between the seven predictive scores for breast cancer (Upper Triangle) and lung cancer (Lower Triangle). Table S5. Breast cancer candidate driver genes predicted by one or more permutation models. Table S6. $P$-values for each permutation model for the 32 breast cancer genes in Cancer Genes Census. Table S7. Lung cancer candidate driver genes predicted by one or more permutation models. Table S8. $P$-values for each permutation model for the 12 lung cancer genes in Cancer Genes Census. (PDF 1721 kb)

Additional file 2: Table S3. Predicted breast cancer driver genes by the seven permutation models. Table S4. Predicted lung cancer driver genes by the seven permutation models. (XLSX $43 \mathrm{~kb}$ )

\section{Abbreviations}

CADD: Combined Annotation Dependent Depletion; CDGs: Cancer Driver Genes; CGC: Cancer Gene Census; DNN: Deep Neural Network; FathmmMKL: Functional Analysis through Hidden Markov Models - Multiple Kernel Learning; InVeX: Introns Vs Exons; LR: Logistic Regression; MDS: Most Deleterious Score; MKL: Multiple Kernel Learning; SMDS: Sum of Most Deleterious Score; SNVs: Single Nucleotide Variants; SVM: Support Vector Machine; UPGMA: Unweighted Pair Group Method with Arithmetic Mean; VEP: Ensembl Variant Effect Predictor; VEST3: Variant Effect Scoring Tool; WES: Whole-Exome Sequencing; WGS: Whole-Genome Sequencing; WGSA: Whole Genome Sequencing Annotator

\section{Acknowledgements}

We thank Mr. Walker Hale IV, Ms. Lauren Petty and Dr. Chang Li for helping with part of programming.

\section{Funding}

Publication charges for this article have been funded by XL's startup funding from the University of South Florida.

\section{Availability of data and materials}

All data used in this manuscript were downloaded from public repositories. Please see the section Cancer Mutation Data collection under Methods.

\section{About this supplement}

This article has been published as part of BMC Medical Genomics Volume 12 Supplement 1, 2019: Selected articles from the International Conference on Intelligent Biology and Medicine (ICIBM) 2018: medical genomics. The full contents of the supplement are available online at https:// bmcmedgenomics.biomedcentral.com/articles/supplements/volume-12supplement-1.

\section{Authors' contributions}

$\mathrm{XL}$ designed the method study and supervised the project. ADN lead the implementation of the method, performed the data analysis and drafted the manuscript. KC helped with the interpretation, and description of the results. All authors read and approved the final manuscript.

\section{Ethics approval and consent to participate}

Not applicable.

\section{Consent for publication}

Not applicable.

\section{Competing interests}

The authors declare that they have no competing interests.

\section{Publisher's Note}

Springer Nature remains neutral with regard to jurisdictional claims in published maps and institutional affiliations.

\section{Author details}

${ }^{1}$ Human Genetics Center, UTHealth School of Public Health, Houston, TX, USA. ${ }^{2}$ Department of Bioinformatics and Computational Biology, The University of Texas MD Anderson Cancer Center, Houston, TX, USA. ${ }^{3}$ Present Address: USF Genomics, College of Public Health, University of South Florida, Tampa, FL, USA.

Published: 31 January 2019

\section{References}

1. Djotsa Nono AB, Chen K, Liu X. Computational prediction of genetic drivers in cancer. In: eLS. Chichester: Wiley; 2016. p. 1-16. https://doi.org/10.1002/ 9780470015902.a0025331.

2. Futreal PA, Coin L, Marshall M, Down T, Hubbard T, Wooster R, Rahman N, Stratton MR. A census of human cancer genes. Nat Rev Cancer. 2004;4(3): 177-83.

3. Forbes $S A$, Beare D, Gunasekaran P, Leung K, Bindal N, Boutselakis $H$, Ding M, Bamford S, Cole C, Ward S, Kok CY, Jia M, De T, Teaque JW, Stratton MR, McDermott U, Campbell PJ. COSMIC: exploring the world's knowledge of somatic mutations in human cancer. Nucleic Acids Res. 2015;43(Database issue):D805-11.

4. Hodis E, Watson IR, Kryukov GV, Arold ST, Imielinski M, Theurillat JP, Nickerson E, Auclair D, Li L, Place C, Dicara D, Ramos AH, Lawrence MS, Cibulskis K, Sivachenko A, Voet D, Saksena G, Stransky N, Onofrio RC, Winckler W, Ardlie K, Wagle N, Wargo J, Chong K, Morton DL, Stemke-Hale K, Chen G, Noble M, Meyerson M, Ladbury JE, Davies MA, Gershenwald JE, Wagner SN, Hoon DS, Schadendorf D, Lander ES, Gabriel SB, Getz G, Garraway LA, Chin L. A landscape of driver mutations in melanoma. Cell. 2012;150(2):251-63.

5. Adzhubei IA, Schmidt S, Peshkin L, Ramensky VE, Gerasimova A, Bork P, Kondrashov AS, Sunyaev SR. A method and server for predicting damaging missense mutations. Nat Methods. 2010;7(4):248-9.

6. Alexandrov LB, Nik-Zainal S, Wedge DC, Aparicio SA, Behjati S, Biankin AV, Bignell GR, Bolli N, Borg A, Børresen-Dale AL, Boyault S, Burkhardt B, Butler AP, Caldas C, Davies HR, Desmedt C, Eils R, Eyfjörd JE, Foekens JA, Greaves M, Hosoda F, Hutter B, llicic T, Imbeaud S, Imielinski M, Jäger N, Jones DT, Jones D, Knappskog S, Kool M, Lakhani SR, López-Otín C, Martin S, Munshi NC, Nakamura H, Northcott PA, Pajic M, Papaemmanuil E, Paradiso A, Pearson JV, Puente XS, Raine K, Ramakrishna M, Richardson AL, Richter J, Rosenstiel P, Schlesner M, Schumacher TN, Span PN, Teague JW, Totoki Y, Tutt AN, Valdés-Mas R, van Buuren MM, Van 't Veer L, Vincent-Salomon A, Waddell N, Yates LR, Australian Pancreatic Cancer Genome Initiative, ICGC Breast Cancer Consortium, ICGC MMML-Seq consortium; ICGC PedBrain, Zucman-Rossi J, Futreal PA, McDermott U, Lichter P, Meyerson M, Grimmond SM, Siebert R, Campo E, Shibata T, Pfister SM, Campbell PJ, Stratton MR. Signatures of mutational processes in human cancer. Nature. 2013;500(7463):415-21. https://doi.org/10.1038/nature12477.

7. Kircher M, Witten DM, Jain P, O'Roak BJ, Cooper GM, Shendure J. A general framework for estimating the relative pathogenicity of human genetic variants. Nat Genet. 2014;46(3):310-5.

8. Quang D, Chen Y, Xie X. DANN: a deep learning approach for annotating the pathogenicity of genetic variants. Bioinformatics. 2015;31(5):761-3.

9. Shihab HA, Rogers MF, Gough J, Mort M, Cooper DN, Day IN, Gaunt TR, Campbell C. An integrative approach to predicting the functional effects of non-coding and coding sequence variation. Bioinformatics. 2015;31(10): $1536-43$.

10. Dong C, Wei P, Jian X, Gibbs R, Boerwinkle E, Wang K, Liu X. Comparison and integration of deleteriousness prediction methods for nonsynonymous SNVs in whole exome sequencing studies. Hum Mol Genet. 2015;24(8):2125-37.

11. Xiong HY, Alipanahi B, Lee LJ, Bretschneider H, Merico D, Yuen RK, Hua Y, Gueroussov S, Najafabadi HS, Hughes TR, Morris Q, Barash Y, Krainer AR, Jojic N, Scherer SW, Blencowe BJ, Frey BJ. RNA splicing. The human splicing code reveals new insights into the genetic determinants of disease. Science. 2015;347(6218):1254806

12. Carter H, Douville C, Stenson PD, Cooper DN, Karchin R. Identifying Mendelian disease genes with the variant effect scoring tool. BMC Genomics. 2013;14(Suppl 3):S3. 
13. Tokheim CJ, Papadopoulos N, Kinzler KW, Vogelstein B, Karchin R. Evaluating the evaluation of cancer driver genes. Proc Natl Acad Sci. 2016;113(50): 14330-5.

14. Li Y, Huang J, Sun J, Xiang S, Yang D, Ying X, Lu M, Li H, Ren G. The transcription levels and prognostic values of seven proteasome alpha subunits in human cancers. Oncotarget. 2017:8(3):4501-19.

15. Liu Y, Liu P, Wen W, James MA, Wang Y, Bailey-Wilson JE, Amos Cl, Pinney SM, Yang P, de Andrade M, Petersen GM, Wiest JS, Fain PR, Schwartz AG, Gazdar A, Gaba C, Rothschild H, Mandal D, Kupert E, Lee J, Seminara D, Minna J, Anderson MW, You M. Haplotype and cell proliferation analyses of candidate lung cancer susceptibility genes on chromosome 15q24-25.1. Cancer Res. 2009;69(19):7844-50.

16. Wang T, Chen T, Thakur A, Liang Y, Gao L, Zhang S, Tian Y, Jin T, Liu JJ, Chen M. Association of PSMA4 polymorphisms with lung cancer susceptibility and response to cisplatin-based chemotherapy in a Chinese Han population. Clin Transl Oncol. 2015;17(7):564-9.

17. Hu XT, Chen W, Wang D, Shi QL, Zhang FB, Liao YQ, Jin M, He C. High expression of proteasome subunit PSMA7 in colorectal cancer is significantly correlated with liver metastasis. Zhonghua Zhong Liu Za Zhi. 2008;30(7):515-8.

18. Greenman C, Stephens P, Smith R, Dalgliesh GL, Hunter C, Bignell G, Davies H, Teague J, Butler A, Stevens C, Edkins S, O'Meara S, Vastrik I, Schmidt EE, Avis T, Barthorpe S, Bhamra G, Buck G, Choudhury B, Clements J, Cole J, Dicks E, Forbes S, Gray K, Halliday K, Harrison R, Hills K, Hinton J, Jenkinson A, Jones D, Menzies A, Mironenko T, Perry J, Raine K, Richardson D, Shepherd R, Small A, Tofts C, Varian J, Webb T, West S, Widaa S, Yates A, Cahill DP, Louis DN, Goldstraw P, Nicholson AG, Brasseur F, Looijenga L, Weber BL, Chiew YE, DeFazio A, Greaves MF, Green AR, Campbell P, Birney E, Easton DF, Chenevix-Trench G, Tan MH, Khoo SK, Teh BT, Yuen ST, Leung SY, Wooster R, Futreal PA, Stratton MR. Patterns of somatic mutation in human cancer genomes. Nature. 2007;446(7132):153-8.

19. Xia J, Jia P, Hutchinson KE, Dahlman KB, Johnson D, Sosman J, Pao W, Zhao Z. A meta-analysis of somatic mutations from next generation sequencing of 241 melanomas: a road map for the study of genes with potential clinical relevance. Mol Cancer Ther. 2014;13(7):1918-28.

20. Oh HR, An CH, Yoo NJ, Lee SH. Frameshift mutations in the mononucleotide repeats of TAF1 and TAF1L genes in gastric and colorectal cancers with regional heterogeneity. Pathol Oncol Res. 2017;23(1):125-30.

21. Stepulak A, Luksch H, Gebhardt C, Uckermann O, Marzahn J, Sifringer M, Rzeski W, Staufner C, Brocke KS, Turski L, Ikonomidou C. Expression of glutamate receptor subunits in human cancers. Histochem Cell Biol. 2009; 132(4):435-45

22. Prickett TD, Samuels Y. Molecular pathways: dysregulated glutamatergic signaling pathways in cancer. Clin Cancer Res. 2012;18(16):4240-6.

23. North WG, Gao G, Memoli VA, Pang RH, Lynch L. Breast cancer expresses functional NMDA receptors. Breast Cancer Res Treat. 2010;122(2):307-14.

24. Ueno K, Hirata H, Hinoda Y, Dahiya R. Frizzled homolog proteins, microRNAs and Wnt signaling in cancer. Int J Cancer. 2013;132(8):1731-40.

25. Wong SC, He CW, Chan CM, Chan AK, Wong HT, Cheung MT, Luk LL, Au TC, Chiu MK, Ma BB, Chan AT. Clinical significance of frizzled homolog 3 protein in colorectal patients. PLoS One. 2013;8(11):e79481.

26. Talukder AK, Agarwal M, Buetow KH, Denèfle PP. Tracking Cancer genetic evolution using OncoTrack. Sci Rep. 2016:6:29647.

27. Liu J, Lee W, Jiang Z, Chen Z, Jhunjhunwala S, Haverty PM, Gnad F, Guan Y, Gilbert HN, Stinson J, Klijn C, Guillory J, Bhatt D, Vartanian S, Walter K, Chan J, Holcomb T, Dijkgraaf P, Johnson S, Koeman J, Minna JD, Gazdar AF, Stern HM, Hoeflich KP, Wu TD, Settleman J, de Sauvage FJ, Gentleman RC, Neve RM, Stokoe D, Modrusan Z, Seshagiri S, Shames DS, Zhang Z. Genome and transcriptome sequencing of lung cancers reveal diverse mutational and splicing events. Genome Res. 2012;22(12):2315-27.

28. Jiao Y, Yonescu R, Offerhaus GJ, Klimstra DS, Maitra A, Eshleman JR, Herman $\mathrm{JG}$, Poh W, Pelosof L, Wolfgang CL, Vogelstein B, Kinzler KW, Hruban RH, Papadopoulos N, Wood LD. Whole-exome sequencing of pancreatic neoplasms with acinar differentiation. J Pathol. 2014;232(4):428-35.

29. Meynet O, Scotlandi K, Pradelli E, Manara MC, Colombo MP, SchmidAntomarchi H, Picci P, Bernard A, Bernard G. Xg expression in Ewing's sarcoma is of prognostic value and contributes to tumor invasiveness. Cancer Res. 2010;70(9):3730-8.

30. Surdez D, Benetkiewicz M, Perrin V, Han ZY, Pierron G, Ballet S, Lamoureux F, Rédini F, Decouvelaere AV, Daudigeos-Dubus E, Geoerger B, de Pinieux G, Delattre O, Tirode F. Targeting the EWSR1-FLI1 oncogene-induced protein kinase PKC- $\beta$ abolishes Ewing sarcoma growth. Cancer Res. 2012;72(17): 4494-503.

31. Harada H, Miyamoto K, Yamashita Y, Taniyama K, Ohdan H, Okada M. Methylated DLX4 predicts response to pathologic stage I non-Small cell lung Cancer resection. Ann Thorac Surg. 2015;99(5):1746-54.

32. Haria D, Trinh BO, Ko SY, Barengo N, Liu J, Naora H. The homeoprotein DLX4 stimulates NF-KB activation and CD44-mediated tumor-mesothelial cell interactions in ovarian cancer. Am J Pathol. 2015;185(8):2298-308.

33. Trinh B, Ko SY, Haria D, Barengo N, Naora H. The homeoprotein DLX4 controls inducible nitric oxide synthase-mediated angiogenesis in ovarian cancer. Mol Cancer. 2015;14:97.

34. Chen YC, Huang RL, Huang YK, Liao YP, Su PH, Wang HC, Chang CC, Lin YW, Yu MH, Chu TY, Lai HC. Methylomics analysis identifies epigenetically silenced genes and implies an activation of $\beta$-catenin signaling in cervical cancer. Int J Cancer. 2014;135(1):117-27.

35. Castellana B, Escuin D, Peiró G, Garcia-Valdecasas B, Vázquez T, Pons C, Pérez Olabarria M, Barnadas A, Lerma E. ASPN and GJB2 are implicated in the mechanisms of invasion of ductal breast carcinomas. J Cancer. 2012;3:175-83.

36. Liang L, Xu J, Wang M, Xu G, Zhang N, Wang G, Zhao Y. LncRNA HCP5 promotes follicular thyroid carcinoma progression via miRNAs sponge. Cell Death Dis. 2018;9(3):372.

37. Shan N, Zhou W, Zhang S, Zhang Y. Identification of HSPA8 as a candidate biomarker for endometrial carcinoma by using iTRAQ-based proteomic analysis. Onco Targets Ther. 2016;9:2169-79.

38. Nibbe RK, Markowitz S, Myeroff L, Ewing R, Chance MR. Discovery and scoring of protein interaction subnetworks discriminative of late stage human colon cancer. Mol Cell Proteomics. 2009;8(4):827-45.

39. Yu X, Feng L, Liu D, Zhang L, Wu B, Jiang W, Han Z, Cheng S. Quantitative proteomics reveals the novel co-expression signatures in early brain development for prognosis of glioblastoma multiforme. Oncotarget. 2016; 7(12):14161-71

40. Kumar P, Henikoff S, Ng PC. Predicting the effects of coding nonsynonymous variants on protein function using the SIFT algorithm. Nat Protoc. 2009:4:1073-81.

41. Davydov EV, Goode DL, Sirota M, Cooper GM, Sidow A, Batzoglou S. Identifying a high fraction of the human genome to be under selective constraint using GERP++. PLoS Comput Biol. 2010;6(12):e1001025.

42. Schwarz JM, Cooper DN, Schuelke M, Seelow D. MutationTaster2: mutation prediction for the deep-sequencing age. Nat Methods. 2014;11(4):361-2.

43. Reva B, Antipin $Y$, Sander C. Predicting the functional impact of protein mutations: application to cancer genomics. Nucleic Acids Res. 2011;39(17): e118

44. Shihab HA, Gough J, Cooper DN, Day IN, Gaunt TR. Predicting the functional consequences of cancer-associated amino acid substitutions. Bioinformatics. 2013:29(12):1504-10.

45. Chun S, Fay JC. Identification of deleterious mutations within three human genomes. Genome Res. 2009;19(9):1553-61.

46. Garber M, Guttman M, Clamp M, Zody MC, Friedman N, Xie X. Identifying novel constrained elements by exploiting biased substitution patterns. Bioinformatics. 2009;25(12):i54-62.

47. Cooper GM, Stone EA, Asimenos G. Distribution and intensity of constraint in mammalian genomic sequence. Genome Res. 2005;15(7):901-13.

48. Carter H, Chen S, Isik L, Tyekucheva S, Velculescu VE, Kinzler KW, Vogelstein B, Karchin R. Cancer-specific high-throughput annotation of somatic mutations: computational prediction of driver missense mutations. Cancer Res. 2009;69(16):6660-7.

49. Liu X, White S, Peng B, Johnson AD, Brody JA, Li AH, Huang Z, Carroll A, We $P$, Gibbs R, Klein RJ, Boerwinkle E. WGSA: an annotation pipeline for human genome sequencing studies. J Med Genet. 2016;53(2):111-2.

50. Wang K, Li M, Hakonarson H. ANNOVAR: functional annotation of genetic variants from high-throughput sequencing data. Nucleic Acids Res. 2010; 38(16):e164.

51. Cingolani P, Platts A, Wang le L, Coon M, Nguyen T, Wang L, Land SJ, Lu X, Ruden DM. A program for annotating and predicting the effects of single nucleotide polymorphisms, SnpEff: SNPs in the genome of Drosophila melanogaster strain w1118; iso-2; iso-3. Fly (Austin). 2012;6(2):80-92.

52. McLaren W, Pritchard B, Rios D, Chen Y, Flicek P, Cunningham F. Deriving the consequences of genomic variants with the Ensembl API and SNP effect predictor. Bioinformatics. 2010;26(16):2069-70.

53. Pruitt KD, Brown GR, Hiatt SM, Thibaud-Nissen F, Astashyn A, Ermolaeva O, Farrell CM, Hart J, Landrum MJ, McGarvey KM, Murphy MR, O'Leary NA, Pujar 
S, Rajput B, Rangwala SH, Riddick LD, Shkeda A, Sun H, Tamez P, Tully RE, Wallin C, Webb D, Weber J, Wu W, DiCuccio M, Kitts P, Maglott DR, Murphy TD, Ostell JM. RefSeq: an update on mammalian reference sequences. Nucleic Acids Res. 2014:42(Database issue):D756-63.

54. Cunningham F, Amode MR, Barrell D, Beal K, Billis K, Brent S, Carvalho-Silva D, Clapham P, Coates G, Fitzgerald S, Gil L, Girón CG, Gordon L, Hourlier T, Hunt SE, Janacek SH, Johnson N, Juettemann T, Kähäri AK, Keenan S, Martin FJ, Maurel T, McLaren W, Murphy DN, Nag R, Overduin B, Parker A, Patricio M, Perry E, Pignatelli M, Riat HS, Sheppard D, Taylor K, Thormann A, Vullo A, Wilder SP, Zadissa A, Aken BL, Birney E, Harrow J, Kinsella R, Muffato M, Ruffier M, Searle SM, Spudich G, Trevanion SJ, Yates A, Zerbino DR, Flicek P. Ensembl 2015. Nucleic Acids Res. 2015;43(Database issue):D662-9.

55. Gjessing HK, Lie RT. Case-parent triads: estimating single- and double-dose effects of fetal and maternal disease gene haplotypes. Ann Hum Genet. 2006;70(Pt 3):382-96.

56. Lex A, Gehlenborg N, Strobelt H, Vuillemot R, Pfister H. UpSet: visualization of intersecting sets. IEEE Trans Vis Comput Graph. 2014;20:1983-92.

Ready to submit your research? Choose BMC and benefit from:

- fast, convenient online submission

- thorough peer review by experienced researchers in your field

- rapid publication on acceptance

- support for research data, including large and complex data types

- gold Open Access which fosters wider collaboration and increased citations

- maximum visibility for your research: over $100 \mathrm{M}$ website views per year

At $\mathrm{BMC}$, research is always in progress.

Learn more biomedcentral.com/submissions 